\title{
FIRST MOTIONS FROM SEISMIC SOURCES NEAR A FREE SURFACE
}

\author{
By R. Burridge, E. R. Lapwood, and L. Knopoff
}

\begin{abstract}
The radiation patterns of first motions are calculated for the sudden occurrence of an arbitrarily oriented fault (dislocation) at the surface of a half space; the dislocation in the fault plane is also arbitrarily oriented and is assumed to occur over a very small area of the fault plane. Initially the source is considered at a finite depth and the solution is obtained by allowing the depth to tend to zero.

In general the results show a surprising directionality for the radiation of $S V$. In the focal plane projection the first motions of $P$ and $S H$ for a strike-slip fault show the familiar fourlobed radiation patterns. The first motions of $S V$ show some reversals in polarity with angular distance from the source.

The first motions for all components of motion for a dip-slip fault have characteristics governed strongly by the presence of the free surface, and hence differ markedly from the usual radiation patterns for a deeply imbedded source.
\end{abstract}

\section{INTRODUCTION}

Theoretical discussions of first motions from seismic sources have usually been based on the assumption that the medium is infinite in all directions, so that the observed disturbances are uninfluenced by the proximity of any boundary.

The first motions from deep foci are not influenced by the presence of a free surface since the phases reflected from the surface arrive late compared with the direct waves. But as the source approaches the surface the direct and reflected phases approach one another in time and an interference can result if observation is made with a long period system. When the source is precisely at the surface, long or short period instruments must record some alterations of the infinite medium radiation pattern in first motions.

In this paper we obtain radiation patterns of first motions when a discontinuity in displacement (a dislocation) occurs suddenly near the surface of a uniform halfspace. We give formulae for the ideal case in which a point source is at zero depth below the surface. These are obtained by a limiting process, and will be good approximations when the linear dimensions of the region of faulting and the depth are small compared with a typical wavelength of the disturbance.

Since even in the simple problem of incidence of a plane wave on a plane surface there is a remarkable variation in amplitude of the reflected waves with angle of incidence, it is to be expected that the proximity of the surface will profoundly modify the radiation pattern. Moreover, when an incident $S$-wave impinges beyond the critical angle, so that there is total reflection, there is a consequent change of phase in the reflected $S$-wave. Thus if there is an incident pulse, which may be regarded as a combination of plane waves, there will be a change of pulse shape on reflection wherever critical reflection beyond the critical angle is involved. Finally, a surface source is associated with head wave effects, not predictable from the infinite medium problem; $S$-wave radiation beyond the critical angle must be influenced by this cause as well. It is the effect of these phenomena that we examine. 
Our sources are arbitrarily oriented dislocations of the type that occur in models of earthquakes. Using the results of Burridge and Knopoff (1964) we obtain equivalent systems of dislocations across the plane $z=h$, the $z$-axis taken normal to the surface, and then solve our boundary value problem in relation to these.
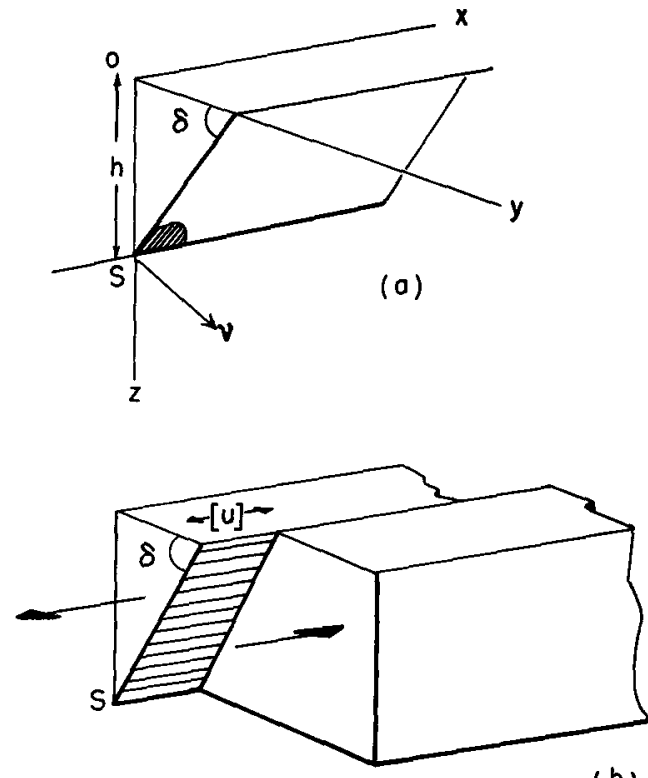

(b)

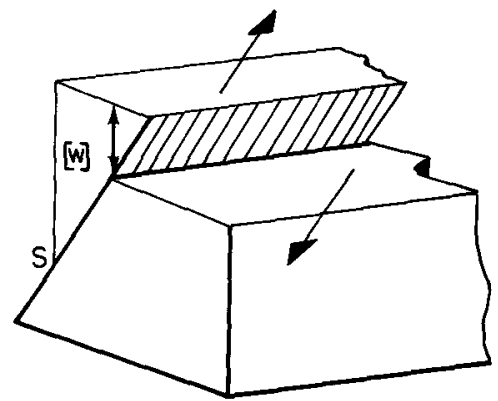

(c)

FIG. 1. (a) Schematic diagram of the free surface $(x O y)$, the source $S$ at depth $h$, and the fault plane dipping at angle $\delta$. (b) A magnified view of the assumed relative motion at the region of faulting (shown hatched in (a)) for a strike-slip fault. (c) The same for a dip-slip fault.

\section{Mathematical Description of Certain Types of Fault, and Equivalent Systems of Point Forces}

We consider models of strike-slip and dip-slip faults on a plane which dips at an angle $\delta$ relative to the plane surface of a half-space. At time $t=0$ the two sides of the fault suddenly acquire a permanent relative displacement tangential to the fault plane; we say that a dislocation occurs. We assume that the normal stresses are continuous across the fault plane.

Let the half-space be defined by $z \geqq 0$ (figure 1 ) and let the faulting take place 
across a small region (shaded in Figure 1a) near the point $(0,0, h)$ of the plane

$$
z-h+y \tan \delta=0
$$

with which we associate the unit normal $v=(0, \sin \delta, \cos \delta) . \delta$ is thus the angle of dip of the fault place, and the strike is parallel to $O x$. We regard the plane $z=0$ as horizontal.

Let the displacement vector be $\mathbf{u}=(u, v, w)$. Then a strike-stip fault is characterized by a discontinuity which we denote by $[u]$ in $u$, while $v$ and $w$ have zero discontinuity (figure 1b). A dip-slip fault is characterized by discontinuities $[v]$ and $[w]$ which are constrained by the relation

$$
[v] \sin \delta+[w] \cos \delta=0
$$

to ensure tangential motions only (Figure 1c).

Taking $r$ and $s$ to be rectangular coordinates in the fault plane such that

$$
x==r, \quad y=s \cos \delta, \quad z=h-s \sin \delta,
$$

we may specify the discontinuities $[u],[v],[w]$ as functions of $r, s$, and time $t$. We then consider the limit as the area of faulting tends to zero at $(0,0, h)$.

I. For a strike-slip dislocation we take

$$
[u]=S \delta(r) \delta(s) H(t)
$$

where $H$ is the Heaviside step-function, $\delta$ the Dirac function, and $S$ is a measure of the amount of offset, which we take as constant. From Burridge and Knopoff (1964) we obtain the equivalent body-force as

$$
\begin{array}{r}
\left(\begin{array}{l}
f_{x} \\
f_{y} \\
f_{z}
\end{array}\right)=S \cos \delta\left(\begin{array}{c}
-\mu \delta(x) \delta(y) \delta^{\prime}(z-h) H(t) \\
0 \\
-\mu \delta^{\prime}(x) \delta(y) \delta(z-h) H(t)
\end{array}\right) \\
+S \sin \delta\left(\begin{array}{c}
-\mu \delta(x) \delta^{\prime}(y) \delta(z-h) H(t) \\
-\mu \delta^{\prime}(x) \delta(y) \delta(z-h) H(t) \\
0
\end{array}\right)
\end{array}
$$

where $\delta^{\prime}(x)=d \delta(x) / d x$ and $\mu$ is the rigidity of the medium.

Taking $\delta=0$ in (2.1), we see that the coefficient of $\cos \delta$ is the body force equivalent of slip on a horizontal plane. This model is denoted by (a). Taking $\delta=90^{\circ}$ we see that the coefficient of $\sin \delta$ is the equivalent of strike-slip on a vertical plane; this model is called (b).

II. For a dip-slip dislocation we take

$$
\begin{aligned}
{[v] } & =-D \delta(r) \delta(s) H(t) \cos \delta \\
{[w] } & =D \delta(r) \delta(s) H(t) \sin \delta
\end{aligned}
$$


where $D$ is constant. The equivalent body force is

$$
\begin{aligned}
\left(\begin{array}{l}
f_{x} \\
f_{y} \\
f_{z}
\end{array}\right)=D \cos 2 \delta\left(\begin{array}{c}
0 \\
-\mu \delta(x) \delta(y) \delta^{\prime}(z-h) H(t) \\
-\mu \delta(x) \delta^{\prime}(y) \delta(z-h) H(t)
\end{array}\right) \\
+D \sin 2 \delta\left(\begin{array}{c}
0 \\
\mu \delta(x) \delta^{\prime}(y) \delta(z-h) H(t) \\
-\mu \delta(x) \delta(y) \delta^{\prime}(z-h) H(t)
\end{array}\right)
\end{aligned}
$$

We observe that the coefficient of $D \cos 2 \delta$, which gives the body force corresponding to unit slip on a horizontal plane, differs from (a) of I only in the interchange of $x$ and $y$. It needs no separate discussion. The coefficient of $\sin 2 \delta$, which is the body force equivalent to dip-slip on a plane dipping at $45^{\circ}$, we denote by (c).

The radiation from dislocations on a plane dipping at angle $\delta$ can thus be expressed as a linear combination of elementary solutions to problems describing the radiation from dislocations on horizontal, vertical, and $45^{\circ}$ planes. To facilitate the solution of the problem with a horizontal boundary, we make further transformations needed to express the prescribed dislocations in terms of dislocations across a horizontal plane only. We consider each of the elementary models in turn.

(a). This body force corresponds to the discontinuity $[u]=S \delta(x) \delta(y) H(t)$ across $z=h$ where the other components of displacement and the stresses are continuous. These six conditions are most conveniently expressed as

$$
\begin{array}{lll}
{[u]=S \delta(x) \delta(y) H(t),} & {\left[\frac{\partial u}{\partial z}\right]=0,} \\
{[v]=0,} & {\left[\frac{\partial v}{\partial z}\right]=0,} \\
{[w]=0,} & {\left[\frac{\partial w}{\partial z}\right]=-\frac{S \lambda}{\lambda+2 \mu} \delta^{\prime}(x) \delta(y) H(t)}
\end{array}
$$

where [ ] denotes the saltus in the direction of positive $z$ across $z=h$, and $\lambda, \mu$ are the Lamé constants of the medium.

(b). This body force, which was derived as equivalent to a strike-slip on a vertical plane, is equivalent also to the following set of discontinuities across $z=h$ (Burridge and Knopoff, loc. cit., formula (10)):

$$
\begin{array}{ll}
{[u]=0,} & {\left[\frac{\partial u}{\partial z}\right]=S \delta(x) \delta^{\prime}(y) H(t),} \\
{[v]=0,} & {\left[\frac{\partial v}{\partial z}\right]=S \delta^{\prime}(x) \delta(y) H(t),} \\
{[w]=0,} & {\left[\frac{\partial w}{\partial z}\right]=0 .}
\end{array}
$$


[Note that since all systems of dislocations that are equivalent to the same body force give the same radiation pattern, it is possible for two different sets of dislocations to give identical radiation patterns, as is well known from fault plane solutions.]

(c). This body force, derived as equivalent to a dip-slip on a plane dipping at $45^{\circ}$ is also equivalent to the set of discontinuities across $z=h$ :

$$
\begin{array}{ll}
{[u]=0,} & {\left[\frac{\partial u}{\partial z}\right]=-\frac{\lambda+\mu}{\lambda+2 \mu} \delta^{\prime}(x) \delta(y) H(t),} \\
{[v]=0,} & {\left[\frac{\partial v}{\partial z}\right]=-\frac{2 \lambda+3 \mu}{\lambda+2 \mu} \delta(x) \delta^{\prime}(y) H(t),} \\
{[w]=\frac{D \mu}{\lambda+2 \mu} \delta(x) \delta(y) H(t),} & {\left[\frac{\partial w}{\partial z}\right]=0 .}
\end{array}
$$

\section{The Fourier Synthesis}

For each of the sources (a), (b) and (c) the values of $[u],[v],[w],[\partial u / \partial z],[\partial v / \partial z]$, $[\partial w / \partial z]$ are given. Using the Fourier inversion formula we may write

$$
[\mathbf{u}]=\frac{1}{(2 \pi)^{3}} \int_{-\infty}^{\infty} d \omega \int_{-\infty}^{\infty} d \xi \int_{-\infty}^{\infty} d \eta[\overline{\mathbf{u}}] e^{i(\omega t-\xi x-\eta \eta)}
$$

where

$$
[\overline{\mathbf{u}}]=\int_{-\infty}^{\infty} d t \int_{-\infty}^{\infty} d x \int_{-\infty}^{\infty} d y[\mathbf{u}] e^{-i(\omega t-\xi x-\eta y)}
$$

Equation (3.1) represents [u] as the result of compounding discontinuities with simple harmonic dependence on $x, y$ and $t$. We can therefore find first the radiation pattern set up when the discontinuities vary harmonically with $x, y$ and $t$, and then combine the results by means of the integrals shown in (3.1) to obtain the radiation due to the originally prescribed discontinuities. We do this because we may expect the solution of the elastic wave equations for harmonically varying discontinuities at $z=h$ to be in terms of plane harmonic or inhomogeneous waves of the types $P$, $S V$, and $S H$.

\section{The Source Functions}

We now construct for the displacement $(u, v, w)$ a sum of solutions of the elastic wave equations which can be made to possess prescribed discontinuities at $z=h$. Consider

$$
\left(\begin{array}{l}
u \\
v \\
w
\end{array}\right)=\left(\begin{array}{l}
\left\{\frac{1}{2} A+\frac{1}{2} A^{\prime} \operatorname{sgn}(z-h)\right\} \xi \\
\left\{\frac{1}{2} A+\frac{1}{2} A^{\prime} \operatorname{sgn}(z-h)\right\} \eta \\
\left\{\frac{1}{2} A \operatorname{sgn}(z-h)+\frac{1}{2} A^{\prime}\right\} \zeta
\end{array}\right)_{P} e^{i(\omega t-\xi x-\eta y-\zeta|z-h|)}+
$$




$$
\begin{gathered}
+\left(\begin{array}{c}
\left\{\frac{1}{2} B+\frac{1}{2} B^{\prime} \operatorname{sgn}(z-h)\right\} \zeta^{\prime} \xi \\
\left\{\frac{1}{2} B+\frac{1}{2} B^{\prime} \operatorname{sgn}(z-h)\right\} \zeta^{\prime} \eta \\
\left\{\frac{1}{2} B \operatorname{sgn}(z-h)+\frac{1}{2} B^{\prime}\right\}\left(-\xi^{2}-\eta^{2}\right)
\end{array}\right)_{S V} e^{i\left(\omega t-\xi x-\eta y-\zeta^{\prime}|z-h|\right)} \\
+\left(\begin{array}{c}
-\left\{\frac{1}{2} C+\frac{1}{2} C^{\prime} \operatorname{sgn}(z-h)\right\} \eta \\
\left\{\frac{1}{2} C+\frac{1}{2} C^{\prime} \operatorname{sgn}(z-h)\right\} \xi \\
0
\end{array}\right)_{S H} e^{i\left(\omega t-\xi x-\eta y-\zeta^{\prime}|z-h|\right)},
\end{gathered}
$$

where $\xi^{2}+\eta^{2}+\zeta^{2}=\omega^{2} / \alpha^{2}, \xi^{2}+\eta^{2}+\zeta^{\prime 2}=\omega^{2} / \beta^{2}, \alpha$ and $\beta$ being the velocities of $P$ and $S$ waves, $\{(\lambda+2 \mu) / \rho\}^{1 / 2}$ and $\{\mu / \rho\}^{1 / 2}$ respectively. $\zeta$ and $\zeta^{\prime}$ are defined as being real and positive or pure imaginary with negative imaginary part: then the corresponding expressions represent plane waves or bounded inhomogeneous waves respectively.

The subscripts $P, S V$ and $S H$ indicate that the forms of the three expressions on the right hand side of (4.1) have been constructed so as to represent waves of the usual types. The combinations of constants $A, B, C, A^{\prime}, B^{\prime}, C^{\prime}$, are so chosen that the terms in $A, B, C$ have discontinuous $z$-derivatives across $z=h$, being themselves continuous, while the terms in $A^{\prime}, B^{\prime}, C^{\prime}$ are discontinuous but have continuous $z$ derivatives.

Since the dislocations will be specified, we can find $A, B, C, A^{\prime}, B^{\prime}, C^{\prime}$ from the following set of equations:

$$
\begin{aligned}
\xi A^{\prime}+\zeta^{\prime} \xi B^{\prime}-\eta C & =[\bar{u}], \\
\eta A^{\prime}+\zeta^{\prime} \eta B^{\prime}+\zeta C & =[\bar{v}], \\
\zeta A-\left(\xi^{2}+\eta^{2}\right) B & =[\bar{w}], \\
-i \zeta \xi A-i \zeta^{\prime 2} \xi B+i \zeta^{\prime} \eta C & =\left[\overline{\frac{\partial u}{\partial z}}\right], \\
-i \zeta \eta A-i \zeta^{\prime 2} \eta B-i \zeta^{\prime} \xi C & =\left[\frac{\partial v}{\partial z}\right], \\
-i \zeta^{2} A^{\prime}+i \zeta^{\prime}\left(\xi^{2}+\eta^{2}\right) B^{\prime} & =\left[\frac{\partial w}{\partial z}\right] .
\end{aligned}
$$

Here we have found the relations for dislocations $[\overline{\mathbf{u}}] e^{i(\omega t-\xi x-\eta y)}$ of (3.1) which form a source varying harmonically. We will subsequently introduce particular values of $[\bar{u}]$, etc. corresponding to source (a), (b) and (c), solve (4.2) for $A, B, C, A^{\prime}, B^{\prime}, C^{\prime}$, and then complete the construction of our solution by integration over $\xi, \eta, \omega$.

\section{Reflections at the Free Surface}

Maintaining the generality provided by the source, which is represented by (4.1), we now seek to satisfy the condition that the boundary $z=0$ is free from stress. 
From our knowledge of reflection of plane waves we expect that incident $P$ will give rise to reflected $P P$ and $P S$, incident $S V$ to reflected $S P$ and $S S V$, incident $S H$ to reflected $S S H$. Using (4.1) as applied to the region $0 \leqq z<h$ and formulating the exponents in the reflected waves according to Snell's Law, we determine the coefficients so that the stresses

$$
\begin{aligned}
\tau_{z x} & =\mu\left(\frac{\partial u}{\partial z}+\frac{\partial w}{\partial x}\right) \\
\tau_{z y} & =\mu\left(\frac{\partial v}{\partial z}+\frac{\partial w}{\partial y}\right) \\
\tau_{z z} & =\lambda\left(\frac{\partial u}{\partial x}+\frac{\partial v}{\partial y}+\frac{\partial w}{\partial z}\right)+2 \mu \frac{\partial w}{\partial z}
\end{aligned}
$$

all vanish on $z=0$. We obtain the complete set of incident and reflected plane waves for which the expressions valid in the region $z>h$ are:

Incident:

$$
\begin{aligned}
& \left(\begin{array}{l}
u \\
v \\
w
\end{array}\right)_{P}=\frac{1}{2}\left(A+A^{\prime}\right)\left(\begin{array}{l}
\xi \\
\eta \\
\zeta
\end{array}\right) e^{i(\omega t-\xi x-\eta y-\zeta(z-h))}, \\
& \left(\begin{array}{l}
u \\
v \\
w
\end{array}\right)_{S V}=\frac{1}{2}\left(B+B^{\prime}\right)\left(\begin{array}{c}
\zeta^{\prime} \xi \\
\zeta^{\prime} \eta \\
-\xi^{2}-\eta^{2}
\end{array}\right) e^{i\left(\omega t-\xi x-\eta y-\zeta^{\prime}(z-h)\right)}, \\
& \left(\begin{array}{l}
u \\
v \\
w
\end{array}\right)_{S H}=\frac{1}{2}\left(C+C^{\prime}\right)\left(\begin{array}{c}
-\eta \\
\xi \\
0
\end{array}\right) e^{i\left(\omega t-\xi x-\eta y-\zeta^{\prime}(z-h)\right)},
\end{aligned}
$$

Reflected:

$$
\begin{gathered}
\left(\begin{array}{l}
u \\
v \\
w
\end{array}\right)_{P P}=-\frac{1}{2}\left(A-A^{\prime}\right) \frac{\Omega^{*}}{R}\left(\begin{array}{l}
\xi \\
\eta \\
\zeta
\end{array}\right) e^{i(\omega t-\xi x-\eta y-\zeta(z+h))}, \\
\left(\begin{array}{l}
u \\
v \\
w
\end{array}\right)_{P S}=\left(A-A^{\prime}\right) \frac{\zeta\left(\frac{\omega^{2}}{2 \beta^{2}}-\xi^{2}-\eta^{2}\right)}{R}\left(\begin{array}{c}
\xi \zeta^{\prime} \\
\eta \zeta^{\prime} \\
-\xi^{2}-\eta^{2}
\end{array}\right) e^{i\left(\omega t-\xi x-\eta y-\zeta^{\prime} z-\xi^{\prime} h\right)}, \\
\left(\begin{array}{l}
u \\
v \\
w
\end{array}\right)_{S P}=\left(B-B^{\prime}\right) \frac{\zeta^{\prime}\left(\xi^{2}+\eta^{2}\right)\left(\frac{\omega^{2}}{2 \beta^{2}}-\xi^{2}-\eta^{2}\right)}{R}\left(\begin{array}{l}
\xi \\
\eta \\
\zeta
\end{array}\right) e^{i\left(\omega t-\xi x-\eta y-\zeta z-\zeta^{\prime} h\right)},(5.1)
\end{gathered}
$$




$$
\begin{aligned}
& \left(\begin{array}{l}
u \\
v \\
w
\end{array}\right)_{S S V}=\frac{1}{2}\left(B-B^{\prime}\right) \frac{\mathbb{R}^{*}}{\mathrm{Q}}\left(\begin{array}{c}
\xi \zeta^{\prime} \\
\eta \zeta^{\prime} \\
-\xi^{2}-\eta^{2}
\end{array}\right) e^{i\left(\omega t-\xi x-\eta y-\zeta^{\prime}(\varepsilon+h)\right)}, \\
& \left(\begin{array}{l}
u \\
v \\
w
\end{array}\right)_{S S H}=\frac{1}{2}\left(C-C^{\prime}\right)\left(\begin{array}{c}
-\eta \\
\xi \\
0
\end{array}\right) e^{i\left(\omega t-\xi x-\eta y-\zeta^{\prime}(2+h)\right)}
\end{aligned}
$$

where

$$
\mathbb{R}=\left(\frac{\omega^{2}}{2 \beta^{2}}-\xi^{2}-\eta^{2}\right)^{2}+\zeta \zeta^{\prime}\left(\xi^{2}+\eta^{2}\right)
$$

and

$$
\mathbb{R}^{*}=\left(\frac{\omega^{2}}{2 \beta^{2}}-\xi^{2}-\eta^{2}\right)^{2}-\zeta \zeta^{\prime}\left(\xi^{2}+\eta^{2}\right)
$$

We now allow $h$ to tend to zero. Then $P, P P$, and $S P$, having the same exponent and the same direction, combine into a single wave. $S, S S V$, and $P S$ also combine, and so do $S H$ and $S S H$.

We have carried the calculations as far as we can for a general source. We now insert the particular values of $A, B, C, A^{\prime}, B^{\prime}, C^{\prime}$ that relate to our sources (a), (b), and (c).

\section{Source (a): Slip on a Horizontal Plane}

Inserting the discontinuities (2.3) into the Fourier transform (3.2) we obtain

$$
\begin{array}{ll}
{[\bar{u}]=\frac{1}{i \omega} S,} & {\left[\overline{\frac{\partial u}{\partial z}}\right]=0,} \\
{[\bar{v}]=0,} & {\left[\overline{\frac{\partial v}{\partial z}}\right]=0,} \\
{[\bar{w}]=0,} & {\left[\overline{\frac{\partial w}{\partial z}}\right]=\frac{1}{i \omega} S \frac{\lambda}{\lambda+2 \mu} i \xi .}
\end{array}
$$

We will omit the factors $S / i \omega$ and $D / i \omega$ and bring them back at a later stage. Substituting these values into (4.2) and solving we get

$$
\begin{aligned}
& A=0, \quad A^{\prime}=2 \frac{\beta^{2}}{\omega^{2}} \xi, \\
& B=0, \quad B^{\prime}=2 \frac{\beta^{2}}{\omega^{2}} \frac{\xi}{\zeta^{\prime}} \frac{\left(\frac{\omega^{2}}{2 \beta^{2}}-\xi^{2}-\eta^{2}\right)}{\xi^{2}+\eta^{2}}, \\
& C=0, \quad C^{\prime}=-\frac{\eta}{\xi^{2}+\eta^{2}} .
\end{aligned}
$$


With these values, all expressions in (5.1) cancel. The reflected disturbance precisely cancels the incident in the limit when $h \rightarrow 0$. It may also be verified directly using known radiation patterns valid in a whole space and known reflection coefficients that $P, P P$ and $S P$ tend to cancel as $h \rightarrow 0$, as do $S H$ and $S S H$. SV, PSV and $S S V$ are more difficult to treat directly owing to critical reflections.

\section{Source (b): Strike-Slip on a Vertical Plane}

We find the Fourier transforms of the discontinuities (2.4) as

$$
\begin{array}{ll}
{[\bar{u}]=0,} & {\left[\overline{\partial u} \frac{\bar{\partial}}{\partial z}\right]=\frac{S}{i \omega}(-i \eta),} \\
{[\bar{v}]=0,} & {[\overline{\partial v}]=\frac{S}{i \omega}(-i \xi),} \\
{[\bar{w}]=0,} & {\left[\overline{\partial w} \frac{\bar{\partial} z}{\partial z}\right]=0 .}
\end{array}
$$

and the solution of (4.2) as

$$
\begin{array}{ll}
A=2 \frac{\beta^{2}}{\omega^{2}} \frac{\xi \eta}{\zeta}, & A^{\prime}=0 \\
B=2 \frac{\beta^{2}}{\omega^{2}} \frac{\xi \eta}{\xi^{2}+\eta^{2}}, & B^{\prime}=0 \\
C=\frac{\xi^{2}-\eta^{2}}{\xi^{\prime}\left(\xi^{2}+\eta^{2}\right)}, & C^{\prime}=0 .
\end{array}
$$

Putting these values into (5.1) and combining terms we get

$$
\begin{aligned}
& \left(\begin{array}{l}
u \\
v \\
w
\end{array}\right)_{P}=\frac{\xi \eta \zeta^{\prime}}{R}\left(\begin{array}{l}
\xi \\
\eta \\
\zeta
\end{array}\right) e^{i(\omega t-\xi x-\eta y-\zeta z)} \\
& \left(\begin{array}{l}
u \\
v \\
w
\end{array}\right)_{S V}=\frac{\xi \eta}{\Re} \frac{\left(\frac{\omega^{2}}{2 \beta^{2}}-\xi^{2}-\eta^{2}\right)}{\xi^{2}+\eta^{2}}\left(\begin{array}{c}
\xi \zeta^{\prime} \\
\eta \zeta^{\prime} \\
-\eta^{2}-\xi^{2}
\end{array}\right) e^{i\left(\omega t-\xi x-\eta y-\zeta^{\prime} z\right)} \\
& \left(\begin{array}{l}
u \\
v \\
w
\end{array}\right)_{S H}=\frac{\xi^{2}-\eta^{2}}{\zeta^{\prime}\left(\xi^{2}+\eta^{2}\right)}\left(\begin{array}{c}
-\eta \\
\xi \\
0
\end{array}\right) e^{i\left(\omega t-\xi x-\eta y-\zeta^{\prime} z\right)}
\end{aligned}
$$

This is the solution corresponding to a harmonically varying source. Re-inserting the factor $S / i \omega$ and integrating, we obtain the formal solution corresponding to 
the prescribed dislocation:

$$
\begin{aligned}
& \left(\begin{array}{l}
u \\
v \\
w
\end{array}\right)_{P}=\frac{S}{(2 \pi)^{3}} \int_{-\infty}^{\infty} \frac{d \omega}{i \omega} \int_{-\infty}^{\infty} d \xi \int_{-\infty}^{\infty} d \eta \frac{\xi \eta \zeta^{\prime}}{\Omega}\left(\begin{array}{l}
\xi \\
\eta \\
\zeta
\end{array}\right) e^{i(\omega t-\xi x-\eta y-\zeta z)}, \\
& \left(\begin{array}{l}
u \\
v \\
w
\end{array}\right)_{S Y}=\frac{S}{(2 \pi)^{3}} \int_{-\infty}^{\infty} \frac{d \omega}{i \omega} \int_{-\infty}^{\infty} d \xi \int_{-\infty}^{\infty} d \eta \frac{\xi \eta}{\Omega} \frac{\left(\frac{\omega^{2}}{2 \beta^{2}}-\xi^{2}-\eta^{2}\right)}{\xi^{2}+\eta^{2}}\left(\begin{array}{c}
\xi \zeta^{\prime} \\
\eta \zeta^{\prime} \\
-\xi^{2}-\eta^{2}
\end{array}\right) e^{i\left(\omega t-\xi x-\eta y-\zeta^{\prime} z\right)}, \\
& \left(\begin{array}{l}
u \\
v \\
w
\end{array}\right)_{S H}=\frac{S}{(2 \pi)^{3}} \int_{-\infty}^{\infty} \frac{d \omega}{i \omega} \int_{-\infty}^{\infty} d \xi \int_{-\infty}^{\infty} d \eta \frac{\xi^{2}-\eta^{2}}{\xi^{\prime}\left(\xi^{2}+\eta^{2}\right)}\left(\begin{array}{c}
-\eta \\
\xi \\
0
\end{array}\right) e^{i\left(\omega t-\xi x-\eta y-\zeta^{\prime} z\right)} .
\end{aligned}
$$

This is the exact formal solution, but to discover its physical meaning it is necessary to evaluate the integrals. As we wish to find only the pattern of first motions, we take the stationary phase approximation which is derived in Appendix I and exhibited in formulae (I.8). We also introduce a right handed set of unit vectors

$$
\hat{\mathbf{r}}=\left(\begin{array}{c}
\sin \theta \cos \phi \\
\sin \theta \sin \phi \\
\cos \theta
\end{array}\right), \quad \hat{\mathbf{k}}=\left(\begin{array}{c}
\cos \theta \cos \phi \\
\cos \theta \sin \phi \\
-\sin \theta
\end{array}\right), \hat{\mathbf{1}}=\left(\begin{array}{c}
-\sin \phi \\
\cos \phi \\
0
\end{array}\right)
$$

where $x=R \cos \phi \sin \theta, y=R \sin \theta \sin \phi$, and $z=R \cos \theta$. Seen along the ray from the source to the point $(x, y, z), \hat{\mathbf{r}}$ is in the line of sight, $\hat{\mathbf{k}}$ is perpendicular to this and points upwards in a vertical plane, and $\hat{\mathbf{I}}=\hat{\mathbf{r}} \times \hat{\mathbf{k}}$ points to the observer's right.

In the notation of Appendix I, for $\mathbf{u}_{P}$, the radiated $P$-wave

$F(\alpha, \sin \theta \cos \phi, \sin \theta \sin \phi)=\frac{\sin ^{2} \theta\left(\frac{\alpha^{2}}{\beta^{2}}-\sin ^{2} \theta\right)^{1 / 2} \sin \phi \cos \phi}{\left(\frac{\alpha^{2}}{2 \beta^{2}}-\sin ^{2} \theta\right)^{2}+\sin ^{2} \theta \cos \theta\left(\frac{\alpha^{2}}{\beta^{2}}-\sin ^{2} \theta\right)^{1 / 2}} \hat{\mathbf{r}}$

and $F$ is real. Hence

$$
\begin{gathered}
\mathbf{u}_{P}=\frac{1}{2 \pi} \frac{S}{\alpha R} \frac{\sin ^{2} \theta \cos \theta\left(\frac{\alpha^{2}}{\beta^{2}}-\sin ^{2} \theta\right)^{1 / 2}}{\left(\frac{\alpha^{2}}{2 \beta^{2}}-\sin ^{2} \theta\right)^{2}+\sin ^{2} \theta \cos \theta\left(\frac{\alpha^{2}}{\beta^{2}}-\sin ^{2} \theta\right)^{1 / 2}} \\
\cdot \sin \phi \cos \phi \hat{\mathbf{r}} \delta(t-R / \alpha)
\end{gathered}
$$

where $R=\left(x^{2}+y^{2}+z^{2}\right)^{1 / 2}$. 
Similarly, for $\mathbf{u}_{S V}$, the radiated $S V$ wave

$F(\beta, \sin \theta \cos \phi, \sin \theta \sin \phi)=\frac{\sin \theta\left(\frac{1}{2}-\sin ^{2} \theta\right)}{\left(\frac{1}{2}-\sin ^{2} \theta\right)^{2}+\sin ^{2} \theta \cos \theta\left(\frac{\beta^{2}}{\alpha^{2}}-\sin ^{2} \theta\right)^{1 / 2}}$

and is real for $\sin \theta<\beta / \alpha$. Hence

$\cdot \sin \phi \cos \phi \hat{\mathbf{k}}$

$\mathbf{u}_{S V}=\frac{1}{2 \pi} \frac{S}{\beta R} \frac{\sin \theta \cos \theta\left(\frac{1}{2}-\sin ^{2} \theta\right)}{\left(\frac{1}{2}-\sin ^{2} \theta\right)^{2}+\sin ^{2} \theta \cos \theta\left(\frac{\beta^{2}}{\alpha^{2}}-\sin ^{2} \theta\right)^{1 / 2}} \sin \phi \cos \phi \hat{\mathbf{k}} \delta(t-R / \beta)$

when $\theta<\sin ^{-1}(\beta / \alpha)$.

When $\sin \theta>\beta / \alpha, F$ is complex. We separate real and imaginary parts, obtaining

$$
\begin{gathered}
\mathbf{u}_{S V}=\frac{1}{2 \pi} \frac{S}{\beta R} \frac{\sin \theta \cos \theta\left(\frac{1}{2}-\sin ^{2} \theta\right)^{3}}{\left(\frac{1}{2}-\sin ^{2} \theta\right)^{4}+\sin ^{4} \theta \cos ^{2} \theta\left(\sin ^{2} \theta-\frac{\beta^{2}}{\alpha^{2}}\right)} \sin \phi \cos \phi \hat{\mathbf{k}} \delta(t-R / \beta) \\
+\frac{1}{2 \pi} \frac{S}{\beta R} \frac{\sin ^{3} \theta \cos ^{2} \theta\left(\frac{1}{2}-\sin ^{2} \theta\right)\left(\sin ^{2} \theta-\frac{\beta^{2}}{\alpha^{2}}\right)}{\left(\frac{1}{2}-\sin ^{2} \theta\right)^{4}+\sin ^{4} \theta \cos ^{2} \theta\left(\sin ^{2} \theta-\frac{\beta^{2}}{\alpha^{2}}\right)} \sin \phi \cos \phi \hat{\mathbf{k}} \delta^{\prime}(t-R / \beta)
\end{gathered}
$$

when $\theta>\sin ^{-1} \beta / \alpha$.

Thus when $\theta>\sin ^{-1} \beta / \alpha, \mathbf{u}_{S V}$ has a form which is constructed from the $\delta$ function and its allied function $\delta^{\prime}$. Again, for $\mathbf{u}_{S H}, F$ is

$$
F(\beta, \sin \theta \cos \phi, \sin \theta \sin \phi)=\tan \theta \cos 2 \phi \hat{\mathbf{l}}
$$

which is real. Thus

$$
\mathbf{u}_{S H}=\frac{1}{2 \pi} \frac{S}{\beta R} \sin \theta \cos 2 \phi \hat{\mathbf{1}} \delta\left(t-R_{S H} / \beta\right),
$$

If the original discontinuities have time variation $G(t)$ instead of $H(t)$, the above solution will be modified by convolution with $g(t)=d G(t) / d t$. In particular, the first motion (for sharp $G(t)$ ) will have time dependence like $g(t-R / \alpha$ ) for $P$ and $g(t-R / \beta)$ for $S H$ and for $S V$ for $\theta<\sin ^{-1} \beta / \alpha$. But for $\theta>\sin ^{-1}(\beta / \alpha)$, formula (7.11) will be modified by replacing $\delta(t-R / \beta)$ by $g(t-R / \beta)$ and $\delta^{\prime}(t-R / \beta)$ by $g^{\prime}(t-R / \beta)$ where $g^{\prime}$ is the allied function of $g$, given by

$$
g^{\prime}(t)=-\frac{1}{\pi} P-\int_{-\infty}^{\infty} g(t-\tau) \frac{1}{\tau} d \tau,
$$


' $P$-' indicating that the Cauchy principal value is to be taken at the singularity $\tau=0$. The function $-1 / \pi \tau$ is in fact $\delta^{\prime}(\tau)$.

The variation of the amplitude of $P$ with direction is seen to depend on two factors, one a function of the polar angle $\theta$ alone, the other a function of azimuth $\phi$ alone. Computations have been made for a Poisson medium in which $\lambda=\mu, \alpha^{2}=3 \beta^{2}$ and in figure 2 the variation of the amplitude of $P$ with $\theta$ is shown by means of a polar diagram for the range $0 \leqq \theta \leqq 90^{\circ}$. The variation with $\phi$ is shown in figure 3 .

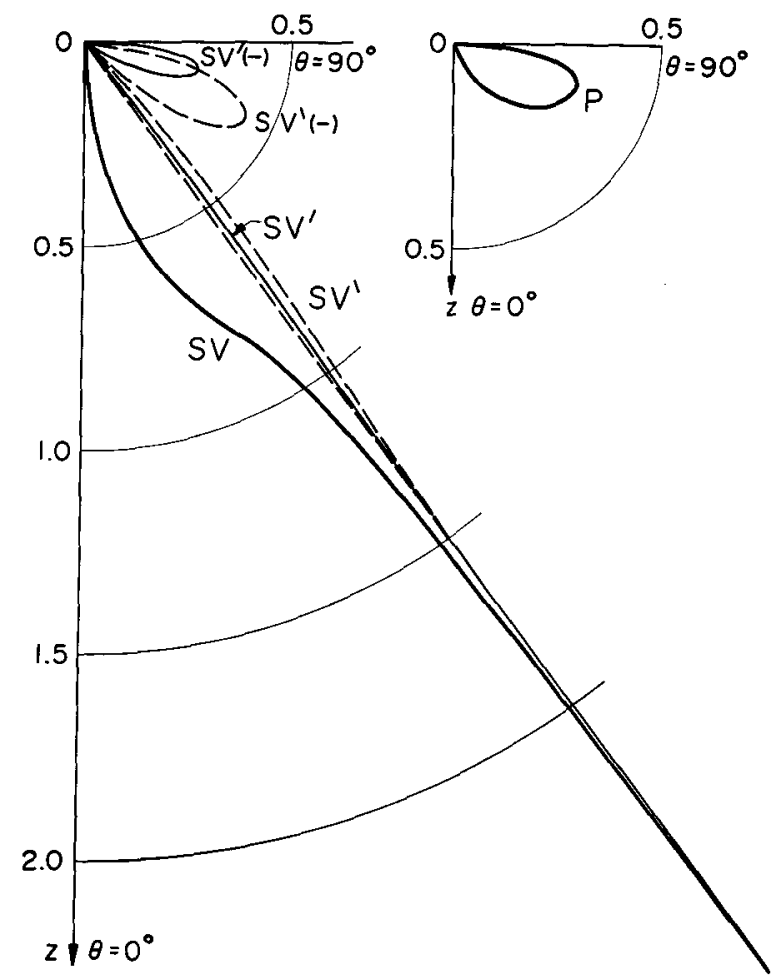

FIG. 2. Polar diagrams for the variation with $\theta$ of $S V,\left(S V^{\prime}\right.$ and $\left.S V^{\prime}\right)$, and $P$ for both the sources (b) and (c).

The amplitude for a given direction $(\theta, \phi)$ is proportional to the product of these two factors. The first motion in $P$ varies with time as $g(t-R / \alpha)$.

The dependence of $S V$ on direction is more complicated, but again variation with $\theta$ and with $\phi$ can be separated. The variation of $S V$ with $\phi$ is the same as that of $P$ and is shown in Figure 3a. The variation with $\theta$ is found as for $P$ as long as $\theta<$ $\sin ^{-1} \beta / \alpha$, and the time dependence is as $f(t-R / \beta)$. But when $\theta>\sin ^{-1} \beta / \alpha S V$ is composed of two terms, namely $S V^{\prime}$ with time variation $g(t-R / \beta)$ together with $S V^{\prime}$ with time variation $g^{\prime}(t-R / \beta) . S V^{\prime}$ and $S V^{\prime}$ are both shown in Figure 2.

The dependence of $S H$ on direction is simple: polar diagrams for the factors $\sin \theta$ and $\cos 2 \phi$ are given in Figures $4 \mathrm{c}$ and $4 \mathrm{a}$ respectively.

In these figures continuous lines indicate positive values, broken lines negative values. 
We observe that the pattern in azimuth is the same for $P$ and $S V$ - the fourlobed rosette shown in figure $3 a$. For $S H$ it is also a four-lobed rosette, but rotated through $45^{\circ}$ compared with that for $P$ and $S V$ (Figure $4 \mathrm{a}$ ).

The most remarkable feature of these graphs is the dramatic variation of $S V, S V^{\prime}$ and $S V^{\prime}$ with $\theta$ near the critical angle $\theta_{c}=\sin ^{-1} \beta / \alpha$. Figures 5 and 6 , in which $\theta$ is

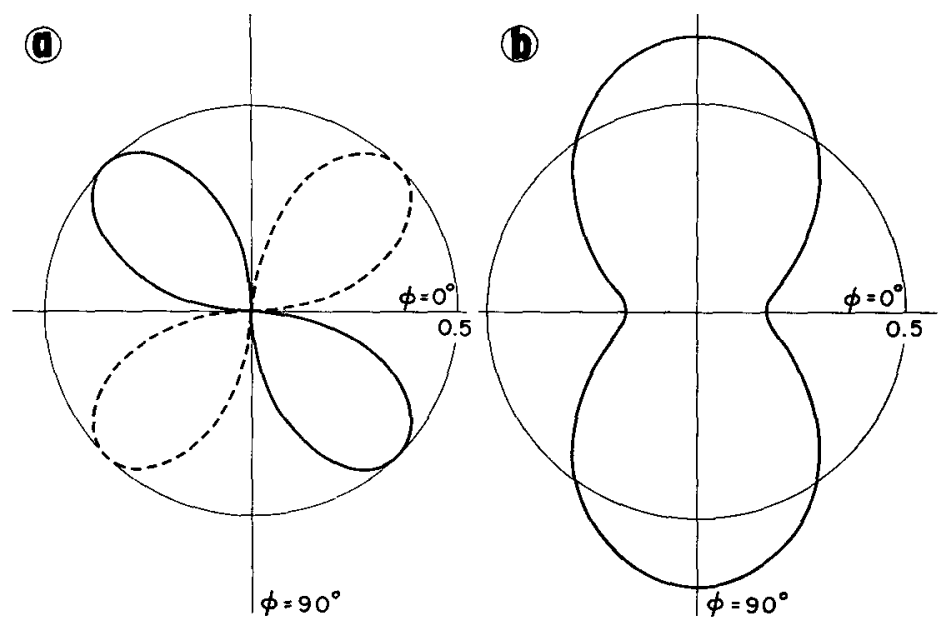

FIG. 3. Polar diagrams for the variation of $P$ and $S V$ with $\phi:$ (a) for the source (b) and (b) for the source $(c)$.

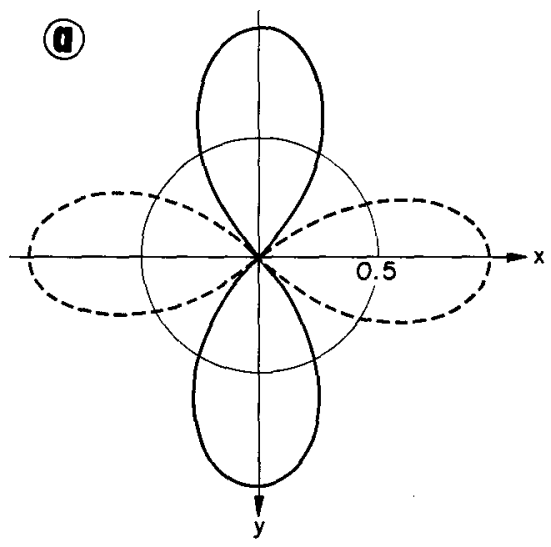

(1)

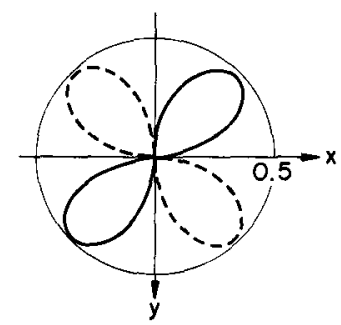

(C)

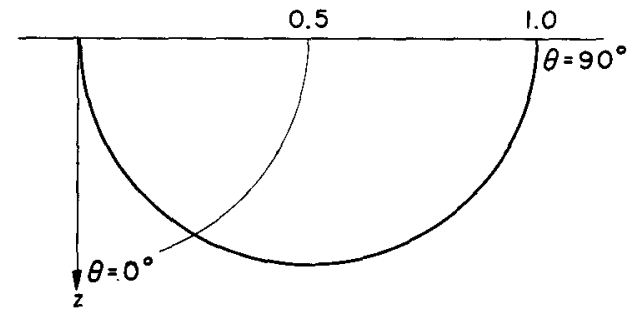

Fig. 4. Polar diagrams for the variation of $S H$ : (a) variation with $\phi$ for source (b), (b) variation with $\phi$ for source (c), (c) variation with $\theta$ common to both sources. 
the abscissa, show this variation. In Figure 6 the horizontal scale is stretched to show detail near $\theta=\theta_{c}$. The steepness of $S V$ to the left and $S V^{\prime}$ to the right of $\theta_{c}$ is due to the vanishing of $\left|\sin ^{2} \theta-\left(\beta^{2} / \alpha^{2}\right)\right|^{1 / 2}$ at $\theta_{c}$, with an infinite derivative there.

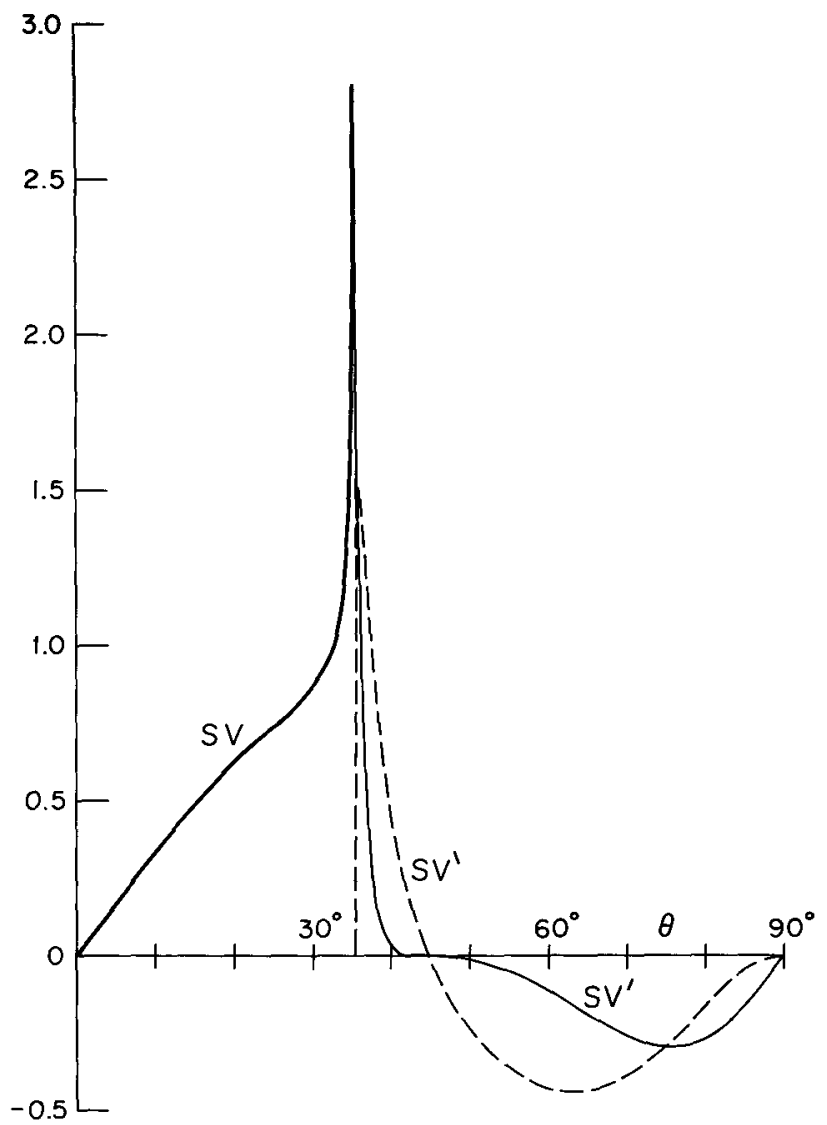

FIG. 5. Linear graph of variation of $S V, S V^{\prime}$ and $S V^{\prime}$ with $\theta$ (cf. Figure 2).

The relative size of the lobes of $P$ and $S V$ in $0<\theta<\theta_{c}$ shows that for such angles most of the radiated energy is in $S V$. This radiation has a large prominence near $\theta_{c}$ showing remarkable sensitivity to direction.

For $\theta>\theta_{c} S V^{\prime}$ is comparable with $S V^{\prime}$ except near $\theta=90^{\circ}$. This means that for a large range of $\theta$ the first motion in $S V$ is a mixture of the original and allied pulses. This in general blunts the sharpness of $S V$ and makes the time of arrival of $S$ hard to identify.

8. Source (c): Dip-Slip on a Plane Dipping at $45^{\circ}$

The Fourier transforms of the discontinuities (2.5) are

$$
\overline{[u]}=0, \quad\left[\frac{\partial u}{\partial z}\right]=\frac{D}{i \omega} \frac{\lambda+\mu}{\lambda+2 \mu} i \xi
$$




$$
\begin{array}{ll}
\overline{[v]}=0, & {\left[\overline{\frac{\partial v}{\partial z}}\right]=\frac{D}{i \omega} \frac{2 \lambda+3 \mu}{\lambda+2 \mu} i \eta,} \\
\overline{[w]}=\frac{D}{i \omega} \frac{\mu}{\lambda+2 \mu}, & {\left[\overline{\frac{\partial w}{\partial z}}\right]=0,}
\end{array}
$$

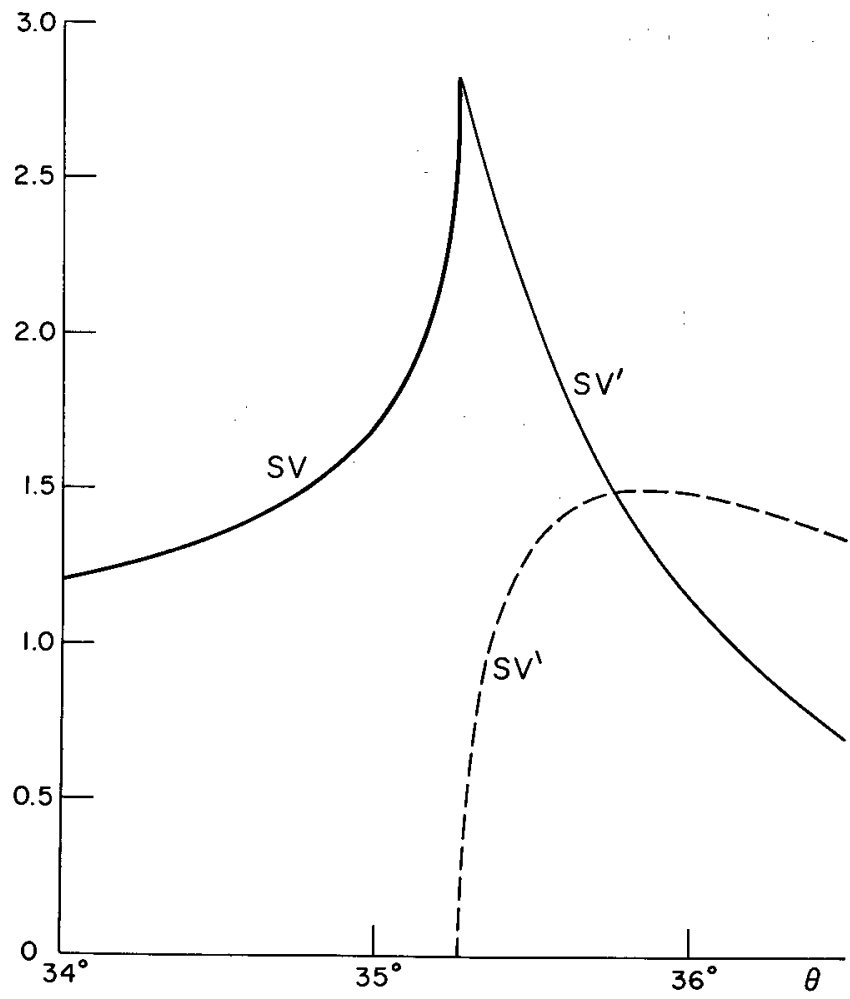

Fig. 6. Magnified detail of Figure 5 near the critical angle $\theta_{c}$.

and the solution of $(4.2)$ is

$$
\begin{array}{ll}
A=-\frac{\beta^{2}}{\omega^{2}} \frac{\eta^{2}-\xi^{2}}{\zeta}, & A^{\prime}=0, \\
B=-\frac{\beta^{2}}{\omega^{2}} \frac{\xi^{2}+2 \eta^{2}}{\xi^{2}+\eta^{2}}, & B^{\prime}=0, \\
C=-\frac{\xi \eta}{\zeta^{\prime}\left(\xi^{2}+\eta^{2}\right)}, & C^{\prime}=0 .
\end{array}
$$

The full formal solution, corresponding to $(7.4),(7.5),(7.6)$ is then

$$
\begin{aligned}
\left(\begin{array}{l}
u \\
v \\
w
\end{array}\right)_{\text {P }}=\frac{-D}{(2 \pi)^{3}} \int_{-\infty}^{\infty} \frac{d \omega}{i \omega} \int_{-\infty}^{\infty} d \xi & \int_{-\infty}^{\infty} d \eta \\
& \cdot\left\{\frac{\lambda}{\lambda+2 \mu}\left(\xi^{2}+\eta^{2}\right)+\eta^{2}\right\} \frac{\zeta^{\prime}}{2 \Omega}\left(\begin{array}{l}
\xi \\
\eta \\
\zeta
\end{array}\right) e^{i(\omega t-\xi x-\eta y-\zeta z)},
\end{aligned}
$$




$$
\begin{aligned}
\left(\begin{array}{l}
u \\
v \\
w
\end{array}\right)_{s v}=\frac{-D}{(2 \pi)^{3}} \int_{-\infty}^{\infty} \frac{d \omega}{i \omega} \int_{-\infty}^{\infty} d \xi \int_{-\infty}^{\infty} d \eta \\
\quad \cdot\left\{\frac{\lambda}{\lambda+2 \mu}\left(\xi^{2}+\eta^{2}\right)+\eta^{2}\right\} \frac{\left(\frac{\omega^{2}}{2 \beta^{2}}-\xi^{2}-\eta^{2}\right)}{2 \mathbb{R}\left(\xi^{2}+\eta^{2}\right)}\left(\begin{array}{c}
\xi \xi^{\prime} \\
\eta \zeta^{\prime} \\
-\xi^{2}-\eta^{2}
\end{array}\right) e^{i\left(\omega t-\xi x-\eta y-\xi^{\prime} z\right)}, \\
\left(\begin{array}{l}
u \\
v \\
w
\end{array}\right)_{S H}=\frac{-D}{(2 \pi)^{3}} \int_{-\infty}^{\infty} \frac{d \omega}{i \omega} \int_{-\infty}^{\infty} d \xi \int_{-\infty}^{\infty} d \eta \frac{1}{\zeta^{\prime}} \frac{\xi \eta}{\xi^{2}+\eta^{2}}\left(\begin{array}{c}
-\eta \\
\xi \\
0
\end{array}\right) e^{i\left(\omega t-\xi x-\eta y^{\prime}-\zeta^{\prime} z\right)},
\end{aligned}
$$

Evaluating the first motion by the method of stationary phase, we obtain, corresponding to $(7.8)-(7.12)$ :

$$
\begin{aligned}
& \mathbf{u}_{P}=-\frac{1}{4 \pi} \frac{D}{\alpha R} \frac{\sin ^{2} \theta \cos \theta\left(\frac{\alpha^{2}}{\beta^{2}}-\sin ^{2} \theta\right)^{1 / 2}\left(\frac{\alpha^{2}}{\beta^{2}}-2+\sin ^{2} \phi\right)}{\left(\frac{\alpha^{2}}{2 \beta^{2}}-\sin ^{2} \theta\right)^{2}+\sin ^{2} \theta \cos \theta\left(\frac{\beta^{2}}{\alpha^{2}}-\sin ^{2} \theta\right)^{1 / 2}} \\
& \mathbf{u}_{S V}=-\frac{1}{4 \pi} \frac{D}{\beta R} \frac{\sin \theta(t-R / \alpha)}{\left(\frac{1}{2}-\sin ^{2} \theta\right)^{2}+\sin ^{2} \theta \cos \theta\left(\frac{\beta^{2}}{\alpha^{2}}-\sin ^{2} \theta\right)^{1 / 2}} \\
& \left.\cdot \hat{\mathbf{k}} \delta(t-R / \beta), \quad \theta<\sin ^{2} \theta\right)\left(\frac{\alpha^{2}}{\beta^{2}}-2+\sin ^{2} \phi\right)
\end{aligned}
$$

and

$$
\begin{array}{r}
\mathbf{u}_{S V}=-\frac{1}{4 \pi} \frac{D}{\beta R} \frac{\sin \theta \cos \theta\left(\frac{1}{2}-\sin ^{2} \theta\right)\left(\frac{\alpha^{2}}{\beta^{2}}-2+\sin ^{2} \phi\right)}{\left(\frac{1}{2}-\sin ^{2} \theta\right)^{4}+\sin ^{4} \theta \cos ^{2} \theta\left(\sin ^{2} \theta-\frac{\beta^{2}}{\alpha^{2}}\right)} \hat{\mathbf{k} \delta}(t-R / \beta) \\
-\frac{1}{4 \pi} \frac{D}{\beta R} \frac{\sin ^{3} \theta \cos ^{2} \theta\left(\frac{1}{2}-\sin ^{2} \theta\right)\left(\sin ^{2} \theta-\frac{\beta^{2}}{\alpha^{2}}\right)^{1 / 2}}{\left(\frac{1}{2}-\sin ^{2} \theta\right)^{4}+\sin ^{4} \theta \cos ^{2} \theta\left(\sin ^{2} \theta-\left(\frac{\beta^{2}}{\alpha^{2}}\right)\right.} \\
\cdot\left(\frac{\alpha^{2}}{\beta^{2}}-2+\sin ^{2} \phi\right) \hat{\mathbf{k}} \delta^{\prime}(t-R / \beta), \theta>\sin ^{-1} \beta / \alpha .
\end{array}
$$

$\mathbf{u}_{S H}=\frac{1}{4 \pi} \frac{D}{\beta R} \sin \theta \sin 2 \phi \hat{\mathbf{l}} \delta(t-R / \beta)$.

It is notable that the variation with $\theta$ of the amplitude of $P, S V$ and $S H$ is the same for source (c) as for source (b). Figures 2 and $4 \mathrm{c}$ therefore represent these 
variations. But the variation with $\phi$ is not the same for both sources. For source (c) $P$ and $S V$ vary with $\phi$ as

$$
\alpha^{2}-2+\sin ^{2} \phi
$$

This is shown in Figure 3b. $S H$ varies as $\sin 2 \phi$ and this is shown in Figure $4 \mathrm{~b}$.

\section{Conclusion}

We have shown that the radiation pattern from a dislocation source of strikeslip or dip-slip type is strongly affected by the proximity of a plane boundary. Peculiarities of reflection lead to rapid variation of amplitude of $S V$ with polar angle, and phase changes, which occur when the polar angle exceeds the critical angle, change the time dependence of the outgoing pulse.

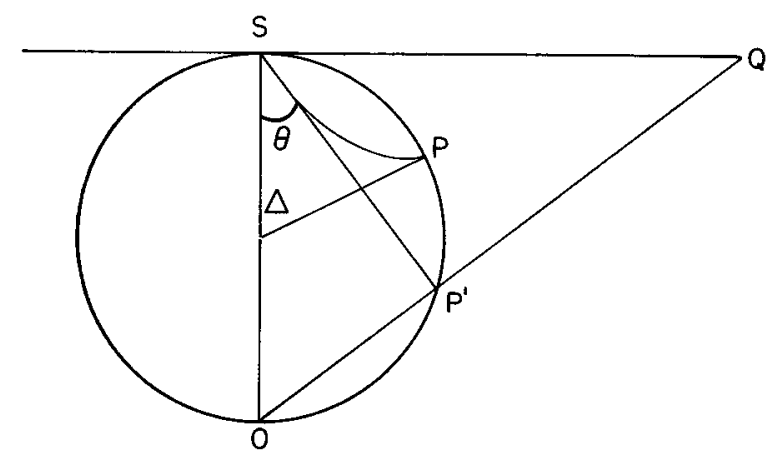

FIG. 7. The plane projection used in Figure 8 and 9 .

It will be observed that only $S H$ has non-zero displacement at a distant point of the free surface. The vanishing of $P$ and $S V$ on $z=0$ is valid for a half-space, but on a spherical surface, this conclusion is not important.

Our solution is a limit as $h \rightarrow 0$. Thus it will be a first approximation to the solution of a problem in which $h$ and the dimensions of the fault are small compared with a typical wavelength of the disturbance. It therefore has most relevance to observations of body waves of long wavelength, but not so long that the high frequency approximation does not hold.

We have given solutions for first motions due to strike-slip on a vertical plane (b) and dip-slip on a fault plane dipping at $45^{\circ}(\mathrm{c})$. If the fault plane dips at an angle $\delta$, our solution for (b) in section 7 must be multiplied throughout by $\sin \delta$ and our solution for (c) in section 8 must be multiplied by $\sin 2 \delta$.

Our calculations have given the first motion radiation pattern from a sudden dislocation occurring near the surface. The general results may be summarized by plotting the expected signs of the first motion on the focal plane projection. The focal plane projection we use (figure 7) projects the intersection of the extended ray with the earth's surface onto the plane tangent at the focus from the antipodal point. By this scheme, observatories near the focus are projected as near the focus 
and observatories at $180^{\circ}$ from the focus are projected at infinity. Thus variation in $\theta$ in our solution appears as a variation in radius in the plane of projection; variation in azimuth $\phi$ appears as a variation in polar angle in the plane.

Using this projection, we can draw the three theoretical patterns representing the radiation expected at a remote observatory, corresponding to each of $P, S V$ and $S H$, for each of the two basic models (Figures 8 and 9 ).
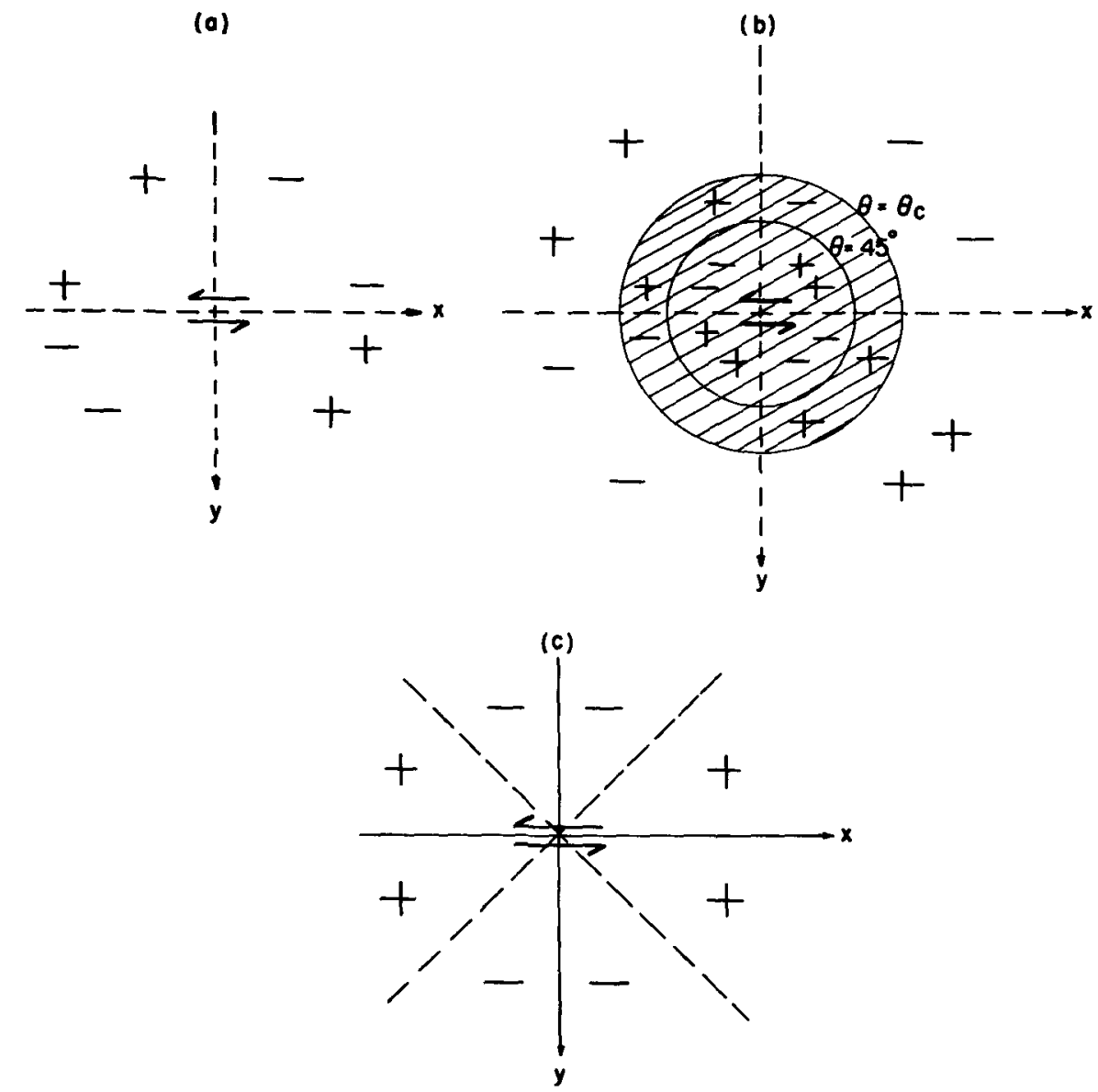

FIG. 8. The signs of first motions corresponding (a) to $P$, (b) to $S V$, (c) to $S H$ for source (b).

In the case of the schematic diagram for the strike-slip motion on a vertical fault plane at the surface (figure 8 ), the radiation in both $P$ and $S H$ is precisely as predicted by the familiar gradient relations obtained for a fault deeply buried beneath the surface. The unusual feature of this solution is, however, the reversal in sign of $S V$ at $\theta=45^{\circ}$. In the earth this corresponds to $\Delta \approx 17 \frac{1}{2}^{\circ}$. The source of this reversal is found in the reversal of the sign of both $S V^{\prime}$ and $S V^{\prime}$ at this angle. Not shown in Figure 8 is the very strong amplitude of $S V$ near the critical angle. The signs of $S V^{\prime}$ and $S V^{\prime}$ are such that no change in sign of the composite $S V$ immediately beyond the critical angle is expected when compared with the $S V$ for angles 
less than critical. The critical angle for a Poisson's ratio of $\frac{1}{4}$ is $\theta \approx 35^{\circ}$, corresponding to $\Delta \approx 23 \frac{1}{2}^{\circ}$. Thus very pronounced amplitudes of $S$ should be observed near $\Delta \approx 23 \frac{1}{2}^{\circ}$. The reversal at $\Delta \approx 17 \frac{1}{2}^{\circ}$ has not been noted in earlier theoretical work on the subject and is strictly the result of interaction with the free surface.

In the case of the schematic diagram for dip-slip faulting, the results are again surprising at first glance. In the first place, the several radiation patterns are identical, except for a multiplicative factor, for all angles of dip of the fault, except for
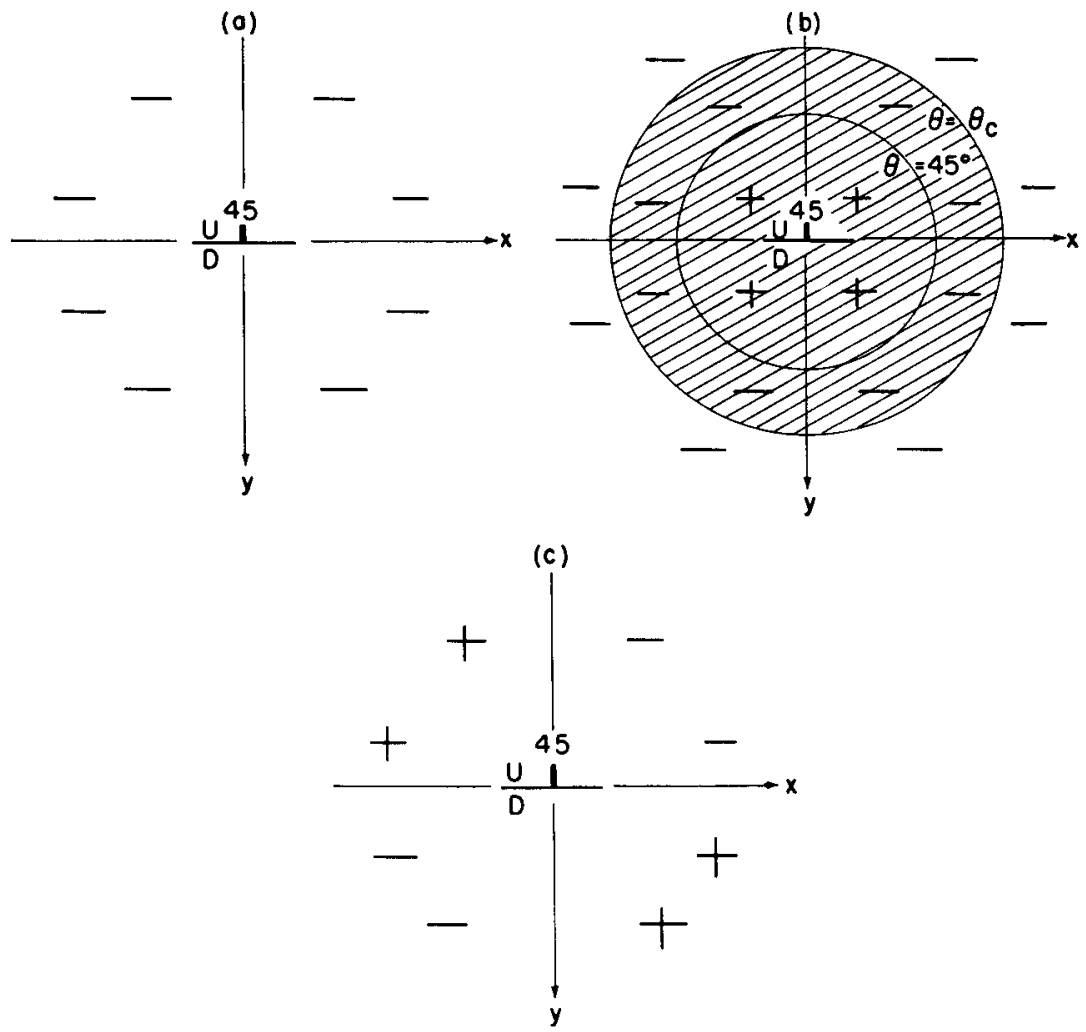

FIG. 9. The signs of first motions corresponding (a) to $P$, (b) to $S V$, (c) to $S H$ for source (c).

the pathological cases of vertical and horizontal faults. This result has been verified in two ways: 1) by the method of this paper, and 2) by considering the $P$ and $S$ radiation from a dislocation in an infinite medium, then considering all the possible reflections from a free surface, and finally adding the incident and reflected rays of the same type (e.g., $P, S P$, and $P P$ ). The second procedure has not been elaborated here.

Since the result for dip-slip faulting is independent of the angle of dip of the fault plane the angular radiation patterns show no dependence upon the dip angle.

The $P$-wave first motions are everywhere of the same sign (figure 9 ); this result represents a significant alteration of the familiar rosette pattern obtained in an infinite medium. The results for $S V$ again show the reversal at $\theta=45^{\circ}$ with a large 
spike in the radiation at the critical angle. The results for $S H$ are unusual since' although the solution has a quadrant organization, the rosette is rotated by $45^{\circ}$ with respect to the solution for the strike-slip fault; in this case a vertical plane normal to the fault is a plane of symmetry, rather than one of antisymmetry.

In figures 8 and 9 our convention has been that positive signs represent compressions in the case of $P$, motions toward the surface in the case of $S V$ and motion in a clockwise direction about the focus in the case of $S H$. A negative sign reverses these directions.

The conclusion that the $P$-wave radiation from a dip-slip motion on a dipping fault is everywhere of the same sign is physically reasonable if one asks how the dip-slip motion originates. In the postulated motions at the fault of figure 9 , the stresses producing the earthquake must have been compressional along the line normal to the strike of the pre-existing fault. Thus, when rupture takes place, the first motion is one of dilatation or motion toward the focus, in agreement with the solution.

The pathological solutions of zero first-motion from dip-slip on a vertical fault should not be considered as requiring that the motion vanishes for all time in a real earthquake. This result only requires that, for this source, the solution vanishes for wavelengths long compared with the vertical extent of the fault. One should obtain a non-vanishing solution for shorter wavelengths in the next order of approximation. This has been carried out in Appendix II and the radiation patterns are given there algebraically.

With regard to the range of applicability of these calculations, we note that a seismograph capable of resolving events 0.1 sec apart should be capable of resolving, in principle, $P$ from $P P$ if the source is more than $250 \mathrm{~m}$ below the surface along the vertical, if we assume crustal $P$ wave velocities of the order of $5 \mathrm{~km} / \mathrm{sec}$. However long period instruments are not capable of performing such a fine resolution and recordings of body wave first motions on long period instruments should be studied carefully.

\section{ACKNOWLEDGMENT}

This research was supported, in part, by research grant No. AF-AFOSR-710-64 of the Air Force Office of Scientific Research as part of the Advanced Research Projects Agency project VELA UNIFORM. One of the authors (E.R.L.) participated in his work as the recipient of an N.S.F. Senior Foreign Scientist Fellowship.

\section{REFERENCE}

Burridge, R. and L. Knopoff

1964. "Body Force Equivalents for Seismic Dislocations," Bull. Seism. Soc. Amer. 54, 19011914. 


\section{Appendix I. Stationary phase approximations for first motion}

In the expressions. (7.4), (7.5), (7.6) and (8.4), (8.5), (8.6), $R$ is an algebraic expression of fourth degree, homogeneous in $\xi, \eta$ and $\omega$. Thus in the integrals with respect to $\xi$ and $\eta$ the integrands are all homogeneous algebraic functions of degree zero multiplied by an exponential. The exponents are all of the type

$$
i\left(\omega t-\xi x-\eta y-\zeta_{0} z\right)
$$

where $\xi^{2}+\eta^{2}+\zeta_{0}{ }^{2}=\omega^{2} / c^{2}$ and $c=\alpha$ or $\beta, \zeta$ being real and positive where $\xi^{2}+\eta^{2}<$ $\omega^{2} / c^{2}$ and $i \zeta_{0}$ real and positive where $\xi^{2}+\eta^{2}>\omega^{2} / c^{2}$.

The method of evaluation of the integrals in (7.4), (7.5), (7.6), (8.3), (8.4), (8.5) will be first to approximate to the integrals with respect to $\xi$ and $\eta$ under the assumption that $\omega$ is large, and then to integrate with respect to $\omega$. The assumption that $|\omega|$ is large is justified by the fact that we are seeking first motions only, and the values of $\omega$ contributing to the first motions are large ones.

Let us therefore consider the integral

$$
I(\omega)=\int_{-\infty}^{\infty} d \xi \int_{-\infty}^{\infty} d \eta F(\omega, \xi, \eta) e^{i\left(-\xi x-\eta y-\zeta_{0} z\right)}
$$

where $F$ is homogeneous of degree zero. We change the variables of integration to $\theta^{\prime}$ and $\phi^{\prime}$ where

$$
\xi=\frac{\omega}{c} \sin \theta^{\prime} \cos \phi^{\prime}, \quad \eta=\frac{\omega}{c} \sin \theta^{\prime} \sin \phi^{\prime}
$$

which implies

$$
\zeta_{0}=\frac{\omega}{c} \cos \theta^{\prime} \quad \text { and } \quad \frac{\partial(\xi, \eta)}{\partial\left(\theta^{\prime}, \phi^{\prime}\right)}=\frac{\omega^{2}}{c^{2}} \sin \theta^{\prime} \cos \theta^{\prime}
$$

If we also express $x, y$, and $z$ in spherical polar coordinates $x=R \sin \theta \cos \phi, y=$ $R \sin \theta \sin \phi, z=R \cos \theta$ we may write

$$
\begin{array}{r}
I(\omega)=\frac{\omega^{2}}{c^{2}} \int_{\Gamma} d \theta^{\prime} \int_{-\pi}^{\pi} d \phi^{\prime} F\left(c, \sin \theta^{\prime} \cos \phi^{\prime}, \sin \theta^{\prime} \sin \phi^{\prime}\right) \sin \theta^{\prime} \cos \theta^{\prime} \\
\cdot e^{-i \frac{\omega R}{c}\left(\sin \theta \sin \theta^{\prime} \cos \left(\phi-\phi^{\prime}\right)+\cos \theta \cos \theta^{\prime}\right)}
\end{array}
$$

where $\Gamma$ is the path 0 to $\frac{1}{2} \pi$ along the real axis and then $\frac{1}{2} \pi$ to $\frac{1}{2} \pi+i \omega$ along a line parallel to the imaginary axis. This ensures that, for $\omega>0,(\omega / c) \cos \theta^{\prime}$ is real and positive or has zero real part and negative imaginary part. If $\omega<0, \Gamma$ is the mirror image of this in the real axis. It is well known that for the purpose of computing first motions only the high frequency components are relevant. Thus we may take $|\omega|$ to be large and treat the integral by Kelvin's method of stationary phase. We shall consider only positive $\omega$ since changing the sign of $\omega$ has the effect of changing 
$I(\omega)$ into its complex conjugate when $\omega$ is real. This is true also for $(1 / i \omega) e^{i \omega t}$ and therefore for the whole expressions in (6.1) and (6.2).

The points of stationary phase (saddle points) for $\phi^{\prime}$ are given by $\sin \theta \sin \theta^{\prime}$. $\sin \left(\phi-\phi^{\prime}\right)=0$ leading to $\phi^{\prime}=\phi$. On setting $\phi^{\prime}=\phi$ the saddle point for $\theta^{\prime}$ is given by $\sin \left(\theta-\theta^{\prime}\right)=0$ leading to $\theta^{\prime}=\theta$. Other saddle points, $\phi^{\prime}=\phi+\pi$ and $\theta^{\prime}=-\theta$ for instance, are not relevant to our purpose since each path of integration may be made to pass through just the one saddle point without changing the value of the integral. We now perform the approximation for the integral with respect to $\phi^{\prime}$ writing $\cos \left(\phi^{\prime}-\phi\right) \approx 1-\frac{1}{2}\left(\phi^{\prime}-\phi\right)^{2}$ when $\phi^{\prime}$ is near $\phi$.

Hence

$I(\omega) \approx \frac{\omega^{2}}{c^{2}} \int_{\Gamma} \sin \theta^{\prime} \cos \theta^{\prime} F\left(c, \sin \theta^{\prime} \cos \phi, \sin \theta^{\prime} \sin \phi\right) e^{-i R_{c}^{\frac{\omega}{\cos }\left(\theta-\theta^{\prime}\right)}} d \theta^{\prime}$

$$
\cdot \int_{-\infty}^{\infty} e^{i \frac{\omega R}{c} \frac{\left(\phi-\phi^{\prime}\right) 2}{2}} \sin \theta \sin \theta^{\prime} d \phi^{\prime}
$$

The inner integral is

$$
\left(\frac{2 \pi c}{R \omega \sin \theta \sin \theta^{\prime}}\right)^{1 / 2} e^{i \pi / 4}
$$

Performing a similar operation for the integration with respect to $\theta^{\prime}$, we obtain $I(\omega) \approx \frac{\omega^{2}}{c^{2}}\left(\frac{2 \pi c}{\omega R \sin ^{2} \theta}\right)^{1 / 2} \sin \theta \cos \theta F(c, \sin \theta \cos \phi, \sin \theta \sin \varphi)$

$$
\begin{gathered}
\cdot e^{-i R \frac{\omega}{c}} e^{i \pi / 4} \int_{-\infty}^{\infty} e^{i \frac{\omega R}{2 c}\left(\theta-\theta^{\prime}\right)^{2}} d \theta^{\prime} \\
=\frac{2 \pi i \omega}{c R} F(c, \sin \theta \cos \phi, \sin \theta \sin \phi) \cos \theta e^{-i \omega R / c} .
\end{gathered}
$$

We will perform one further step before applying the formulae to the special values in sections 7 and 8 . We compute

$$
J(t)=\frac{1}{(2 \pi)^{3}} \int_{-\infty}^{\infty} \frac{1}{i \omega} I(\omega) e^{i \omega t} d \omega
$$

to obtain the pulse shape. Notice that $F(c, \sin \theta \cos \phi, \sin \theta \sin \phi)$ is not a function of $\omega$ and write $F(c, \sin \theta \cos \phi, \sin \theta \sin \phi)=\operatorname{Re} F+i \operatorname{Im} F$. Thus

$$
J(t) \approx \frac{\cos \theta}{\operatorname{Rc}(2 \pi)^{2}} \int_{-\infty}^{\infty} e^{i \omega(t-R / c)}\{\operatorname{Re} F+i \operatorname{Im} F \operatorname{sgn} \omega\} d \omega .
$$

But

$$
\frac{1}{2 \pi} \int_{-\infty}^{\infty} e^{i \omega(t-R / c)} d \omega=\delta(t-R / c)
$$


and

$$
\frac{1}{2 \pi} \int_{-\infty}^{\infty} e^{i \omega(t-R / c)} i \operatorname{sgn} \omega d \omega=\delta^{\prime}(t-R / c)
$$

where $\delta^{\prime}$ is the allied function of $\delta$. Therefore

$$
J(t) \approx \frac{\cos \theta}{2 \pi c R}\left\{\operatorname{Re} F \cdot \delta(t-R / c)+\operatorname{Im} F^{\prime} \cdot \delta^{\prime}(t-R / c)\right\}
$$

Appendix II. A dip-slip on a vertical fault

In our model (a) we found that when we allowed $h \rightarrow 0$ the motion vanished. It would seem sensible, however, to expand the solution in powers of $h$ since in reality every seismic source has some depth even though it might be small. Our result may be interpreted as the vanishing of the term independent of $h$ and we now seek the term in $h$ to the first power. The coefficient of $h$ in this case is what we wish to calculate and to evaluate it we divide by $h$, before allowing $h$ to tend to zero.

Source (a), which has been called the slip on a horizontal plane, gives identical radiation to the source specified by $[w]=S \delta(y) \delta(z-h) H(t)$, the components $u, v$, and the tractions being continuous, the square brackets referring to discontinuities across the plane $x=0$.

On substituting the values of $A, B, C, A^{\prime}, B^{\prime}, C^{\prime}$ given in (6.2) into (5.1) we have

$$
\begin{array}{r}
\left(\begin{array}{l}
u \\
v \\
w
\end{array}\right)_{P}=\frac{\beta^{2}}{\omega^{2}} \frac{\xi}{R}\left[\Re e^{i h \zeta}+\AA^{*} e^{-i h \zeta}-2\left(\frac{\omega^{2}}{2 \beta^{2}}-\xi^{2}-\eta^{2}\right)^{2} e^{-i \zeta^{\prime} h}\right]\left(\begin{array}{l}
\xi \\
\eta \\
\zeta
\end{array}\right) e^{i(\omega t-\xi x-\eta y-\zeta z)} \\
\left(\begin{array}{l}
u \\
v \\
w
\end{array}\right)_{S V}=\frac{\beta^{2}}{\omega^{2}} \frac{\left(\frac{\omega^{2}}{2 \beta^{2}}-\xi^{2}-\eta^{2}\right)}{\left(\xi^{2}+\eta^{2}\right) \Re}\left[\frac{1}{\zeta^{\prime}}\left(R e^{i \zeta^{\prime} h}-\mathbb{R}^{*} e^{-i \zeta^{\prime} h}\right)-2 \zeta\left(\xi^{2}+\eta^{2}\right) e^{-i \zeta h}\right] \\
\cdot\left(\begin{array}{c}
\xi \zeta^{\prime} \\
\eta \zeta^{\prime} \\
-\xi^{2}-\eta^{2}
\end{array}\right) e^{i\left(\omega t-\xi x-\eta y-\zeta^{\prime} z\right)}
\end{array}
$$

$$
\left(\begin{array}{l}
u \\
v \\
w
\end{array}\right)_{S H}=\frac{-\eta}{\xi^{2}+\eta^{2}}\left(e^{i \zeta^{\prime} h}-e^{-i \zeta^{\prime} h}\right)\left(\begin{array}{c}
-\eta \\
\xi \\
0
\end{array}\right) e^{i\left(\omega t-\xi x-\eta y-\zeta^{\prime} z\right)} .
$$

These each tend to zero as $h \rightarrow 0$ but

$$
\lim _{h \rightarrow 0} \frac{1}{h}\left(\begin{array}{l}
u \\
v \\
w
\end{array}\right)_{P}=\frac{2 i \beta^{2}}{\omega^{2}} \frac{\xi \xi^{\prime}}{R}\left[\left(\frac{\omega^{2}}{2 \beta^{2}}-\xi^{2}-\eta^{2}\right)^{2}+\zeta^{2}\left(\xi^{2}+\eta^{2}\right)\right]\left(\begin{array}{l}
\xi \\
\eta \\
\zeta
\end{array}\right) e^{i(\omega t-\xi x-\eta y-\zeta z)}
$$




$$
\begin{aligned}
\lim _{h \rightarrow 0} \frac{1}{h}\left(\begin{array}{l}
u \\
v \\
w
\end{array}\right)_{s V}=\frac{2 i \beta^{2}}{\omega^{2}} \frac{\xi}{\xi^{2}+\eta^{2}} \frac{\left(\frac{\omega^{2}}{2 \beta^{2}}-\xi^{2}-\eta^{2}\right)}{R} & {\left[\left(\frac{\omega^{2}}{2 \beta^{2}}-\xi^{2}-\eta^{2}\right)^{2}+\xi^{2}\left(\xi^{2}+\eta^{2}\right)\right] } \\
& \cdot\left(\begin{array}{c}
\xi \xi^{\prime} \\
\eta \zeta^{\prime} \\
-\xi^{2}-\eta^{2}
\end{array}\right) e^{i\left(\omega t-\xi x-\eta y-\zeta^{\prime} z\right)}
\end{aligned}
$$

$\lim _{h \rightarrow 0} \frac{1}{h}\left(\begin{array}{l}u \\ v \\ w\end{array}\right)_{S H}=\frac{-2 i \zeta^{\prime} \eta}{\xi^{2}+\eta^{2}}\left(\begin{array}{c}-\eta \\ \xi \\ 0\end{array}\right) e^{i\left(\omega t-\xi x-\eta y-\zeta z^{\prime}\right)}$

Each of these is of the form

$$
i \omega F(\omega, \xi, \eta) e^{i\left(-\xi x-\eta y-\zeta_{0} z\right)}
$$

where $F$ is algebraic, homogeneous, and of degree zero in its arguments. We may use the results of Appendix I as far as equation (I.4) but we shall need instead of $J(t)$, (I.5) a function $K(t)$ defined as

$$
\begin{aligned}
K(t) & =\frac{1}{(2 \pi)^{3}} \int_{-\infty}^{\infty} I(\omega) e^{i \omega t} d \omega \\
& =\frac{\cos \theta}{c R(2 \pi)^{2}} \int_{-\infty}^{\infty} i \omega e^{i \omega(t-R / c)}(\operatorname{Re} F+i \operatorname{sgn} \omega \operatorname{Im} F) d \omega \\
& =\frac{\cos \theta}{2 \pi c R}\left\{\operatorname{Re} F \delta^{\prime}(t-R / c)+\operatorname{Im} F\left(\delta^{\prime}\right)^{\prime}(t-R / c)\right\}
\end{aligned}
$$

where $\left(\delta^{\prime}\right)^{\prime}$ is the allied function of $\delta^{\prime}$ and is given explicitly by $\left(\delta^{\prime}\right)^{\prime}(t)=1 / \pi t^{2}$.

Using this result the first motions are given by

$$
\begin{gathered}
\mathbf{u}_{P}=\frac{\beta^{2}}{\pi \alpha^{3}} \frac{S}{R} \frac{\sin \theta \cos \theta\left(\frac{\alpha^{2}}{\beta^{2}}-\sin ^{2} \theta\right)^{1 / 2}\left[\left(\frac{\alpha^{2}}{2 \beta^{2}}-\sin ^{2} \theta\right)^{2}+\sin ^{2} \theta \cos ^{2} \theta\right]}{\left(\frac{\alpha^{2}}{2 \beta^{2}}-\sin ^{2} \theta\right)^{2}+\sin ^{2} \theta \cos \theta\left(\frac{\alpha^{2}}{\beta^{2}}-\sin ^{2} \theta\right)^{1 / 2}} \\
\mathbf{u}_{S V}=\frac{S}{\pi \beta R} \frac{\cos \phi \hat{\mathbf{r}} \delta^{\prime}(t-R / \alpha),}{\left(\frac{1}{2}-\sin ^{2} \theta\right)^{2}+\sin ^{2} \theta \cos \theta\left(\frac{\beta^{2}}{\alpha^{2}}-\sin ^{2} \theta\right)^{1 / 2} \theta\left[\left(\frac{1}{2}-\sin ^{2} \theta\right)^{2}+\sin ^{2} \theta\left(\beta^{2} / \alpha^{2}-\sin ^{2} \theta\right]\right.} \\
\cdot \cos \phi \hat{\mathbf{k}} \delta^{\prime}(t-R / \beta)
\end{gathered}
$$

$\times \theta<\sin ^{-1}(\beta / \alpha)$, 


$$
\begin{aligned}
& \cos \theta\left(\frac{1}{2}-\sin ^{2} \theta\right)^{3}\left[\left(\frac{1}{2}-\sin ^{2} \theta\right)^{2}\right. \\
& \mathbf{u}_{S V}=\frac{S}{R \pi \beta} \frac{\left.+\sin ^{2} \theta\left(\beta^{2} / \alpha^{2}-\sin ^{2} \theta\right)\right] \cos \phi \hat{\mathbf{k}} \delta^{\prime}(t-R / \beta)}{\left(\frac{1}{2}-\sin ^{2} \theta\right)^{4}+\sin ^{4} \theta \cos ^{2} \theta\left(\sin ^{2} \theta-\beta^{2} / \alpha^{2}\right)} \\
& \sin ^{2} \theta \cos ^{2} \theta\left(\sin ^{2} \theta-\beta^{2} / \alpha^{2}\right)^{1 / 2} \\
& -\frac{S}{R \pi \beta} \frac{\cdot\left[\left(\frac{1}{2}-\sin ^{2} \theta\right)^{2}+\sin ^{2} \theta \cos ^{2} \theta\right] \cos \phi \hat{\mathbf{k}}\left(\delta^{\prime}\right)^{\prime}(t-R / \beta)}{\left(\frac{1}{2}-\sin ^{2} \theta\right)^{4}+\sin ^{4} \theta \cos ^{2} \theta\left(\sin ^{2} \theta-\beta^{2} / \alpha^{2}\right)} \\
& \cdot \theta>\sin ^{-1}(\beta / \alpha)
\end{aligned}
$$

$\mathbf{u}_{S H}=-\frac{S}{\pi \beta R} \cos ^{2} \theta \sin \phi \hat{\mathbf{1}} \delta^{\prime}\left(t-R_{0} / \beta\right)$

\section{R. BURRIDGE}

Seismological Laboratory, California Institute of Technology, Pasadena, California.

E. R. LAPWOOD

Department of Mathematies, University of Cambridge, Cambridge, England.

L. KNOPOFF

Institute of Geophysies and Department of Physies, University of California, Los Angeles, California.

Publication No. 394, Institute of Geophysics, University of California, Los Angeles.

Publication No. 1286, Seismological Laboratory, California Institute of Technology, Pasadena.

Manuscript received August 14, 1964. 